\title{
Nickel-organic Frameworks with Hierarchical Flowers Structure Fabricated by Surfactant-assisted Solvothermal Method for High-performance Supercapacitors
}

Yi Wu ${ }^{1}$, Xiangpeng Ding ${ }^{1}$, Yumei Luo ${ }^{1,2}, \mathrm{Fen} \mathrm{X}^{1,2, *}$, Lixian Sun ${ }^{1,2, *}$, JianHao Lao ${ }^{1}$, Xiaohui Qin ${ }^{1}$, Cai Dan $^{1,2,{ }^{*}}, Y u$ Wang $^{1}$, Qingqing Yin ${ }^{1}$, Tao Wang ${ }^{1}$, Kexiang Zhang ${ }^{1,2}$, Bin Li ${ }^{1,2}$, Huanzhi Zhang ${ }^{1,2}$, Yongjin $\mathrm{Zou}^{1,2}$

${ }^{1}$ School of Material Science and Engineering, Guilin University of Electronic Technology, Guilin, 541004, P. R. China.

${ }^{2}$ Guangxi Key Laboratory of Information Materials, Guangxi Collaborative Innovation Center of Structure and Property for New Energy and Materials.

*E-mail: xufen@guet.edu.cn or 1732211579@qq.com, sunlx@ guet.edu.cn, Dancai1985@ guet.edu.cn

doi: $10.20964 / 2021.03 .68$

Received: 17 November 2020 / Accepted: 12 January 2021 / Published: 31 January 2021

In this work, Ni-MOF (R-160) with a hierarchical flower-like structure is fabricated by a facile solvothermal method with surfactant assistance. Benefiting from its structure, R-160 has a very high specific surface area and a large number of exposed active sites. Electrochemical research results show that the electrochemical performance of R-160 prepared with sodium dodecyl sulfate (SDS) is superior to that of B-160 prepared without SDS assistance. For instance, the specific capacitance of B-160 is only $808 \mathrm{~F} \mathrm{~g}^{-1}$ at a current density of $1 \mathrm{~A} \mathrm{~g} \mathrm{~g}^{-1}$. Nevertheless, the specific capacitance of R-160 reaches $1036 \mathrm{~F}$ $\mathrm{g}^{-1}$ under the same conditions, which is $28.2 \%$ higher than that of B-160. Moreover, the energy density of an asymmetric device assembled with R-160 and AC is $38.3 \mathrm{~W} \mathrm{~h} \mathrm{~kg}^{-1}$ at a power density of $749.9 \mathrm{~W}$ $\mathrm{kg}^{-1}$.

Keywords: nickel-metal-organic framework; surfactant; solvothermal method; supercapacitor

\section{FULL TEXT}

(C) 2021 The Authors. Published by ESG (www.electrochemsci.org). This article is an open access article distributed under the terms and conditions of the Creative Commons Attribution license (http://creativecommons.org/licenses/by/4.0/). 